\title{
Age, period and cohort effects and the predictors of physical activity and sedentary behaviour among Chinese children, from 2004 to 2011
}

Xinping Wei ${ }^{1}$, Yu Zang ${ }^{2}$, Xiaodong $\mathrm{Jia}^{3}$, Xiangui He${ }^{4}$, Shurong Zou ${ }^{3}$, Hui Wang ${ }^{5}$, Meihua Shen ${ }^{6,7^{*}}$ and Jiajie Zang ${ }^{3^{*}}$

\begin{abstract}
Background: Very few studies have explored the effects of age, time period, and cohort in association with biological, behavioral, economic, and environmental factors predictors on physical activity (PA) and sedentary behaviour (SB) among Chinese children.

Methods: We used data from a cohort study of the China Health and Nutrition Survey (CHNS) between 2004 and 2011 (2004, 2006, 2009 and 2011). The outcomes of interest were metabolic equivalent of task (MET) hours per week from both active and sedentary activities. Age, gender, individual characteristics, household size, asset ownership, and urbanisation were included as covariates. Age, period and cohort effects analyses for PA and SB of children (6-17 y, $n=3528$ ) was conducted to explicitly assess differences in PA and SB due to age vs. period effects, and implicitly assess differences by cohorts due to the period-specific experiences across individuals of varying ages.

Results: The mean age of the sample in each time point fluctuated from 12.6 to 11.3 years and PA slightly decreased from 50.0 \pm 63.2 MET hours per week (MET-hr./wk) in 2004 to $47.1 \pm 54.9$ MET-hr./wk. in 2011. However, SB increased from $31.8 \pm 22.0$ MET-hr./wk. to $37.6 \pm 22.2 \mathrm{MET}$-hr./wk. Girls had lower PA and higher SB levels than boys. Controlling for age effects, marginal period effects on PA were observed in some survey years. Higher levels of urbanisation and number of household computers served as negative and positive predictors for PA and SB, respectively. Higher household income was a positive predictor of SB. Surprisingly, bigger household size was the only negative predictor of SB $(P<0.05)$.
\end{abstract}

Conclusions: This longitudinal study followed a large cohort of children over a significant period of their childhood. We observed potential age and secular trends in PA levels. Higher community urbanisation and number of home computers were associated with both PA and SB levels. Larger household size was the only factor that was negatively associated with SB. These findings shed light on health policy and preventative health strategies for China and other countries that are now facing similar public health challenges.

Keywords: Physical activity, Age, Period, Cohort, Children, China

\footnotetext{
* Correspondence: littlelovehouse@126.com; zangjiajie@scdc.sh.cn

Xinping Wei, Yu Zang and Xiaodong Jia contributed equally to this work. Jiajie Zang, and Meihua Shen contributed equally.

${ }^{6}$ Department of Intensive Care Unit, Shanghai Provincial Crops Hospital,

Chinese People's Armed Police Forces, 831 Hongxu Road, Shanghai 201103

People's Republic of China

${ }^{3}$ Department of Nutrition Hygiene, Division of Health Risk Factor Monitoring and Control, Shanghai Municipal Center for Disease Control and Prevention 1380 West Zhongshan Road, Changning District, Shanghai 200336, People's Republic of China

Full list of author information is available at the end of the article
} 


\section{Background}

Many studies suggest that physical activity levels have decreased significantly in recent years. A total of only $35.6 \%$ students spent more than $1 \mathrm{~h} /$ day performing moderate to vigorous physical activity (MVPA) during school, and $34.9 \%$ spent more than $1 \mathrm{~h} /$ day performing MVPA outside school hours in Beijing, China [1]. The inexorable rise in obesity has led to widespread calls for regular monitoring of child and adolescent weight in order to counter rising prevalence of obesity worldwide $[2,3]$. Childhood obesity can precipitate adverse health and social consequences such as adult obesity, cardiovascular diseases, and type 2 diabetes mellitus [4]. Since physical activity (PA) contributes towards $18-29 \%$ of daily energy expenditure, it has become an important focus for research into targeting very high rates of childhood obesity [5].

It is widely accepted that engaging in regular physical activity is positively associated with a range of beneficial childhood health and fitness outcomes [6, 7]. Furthermore, it helps reduce the risk of non-communicable diseases (NCDs) in adults such as cardiovascular and cerebrovascular diseases, diabetes, obesity, hypertension, cognitive function and certain cancers $[8,9]$. However, while the benefits of physical activity are well accepted, unprecedented social, economic, and educational system changes in China have been associated with increasingly lower levels of PA and higher levels of SB in Chinese children and adolescents. As economic and technological improvements have facilitated domestic housework and transportation, this has resulted in an increasingly sedentary lifestyle (e.g., watching television, playing video games, studying, and internet browsing) across the country in recent years [10].

There is public health concern that sedentary behaviours among children and adolescents may displace the time available for participation in PA, resulting in overall lower energy expenditure and consequent chronic health outcomes that are independent of PA [11-15]. Hence, it is important to evaluate both PA and SB separately.

Engagement in various types or forms of childhood PA is likely related to age, life-course, socioeconomic factors, asset ownership, the built environment, and degree of urbanisation. Therefore, measurement of these factors is important for increasing our understanding of how changes in PA may alter risk for obesity or disease. However, PA data for children and adolescents available in China are often cross-sectional [16-18]. Indeed, previous studies using such cross-sectional data to study epidemiological associations between exposures and PA between age groups may represent differences between birth cohort years rather than effects of exposures, which can be confounding [16-18]. Although there have been prospective studies of PA in China [19-21], these studies did not assess the period and birth cohort effects over and above age groups [19-21]. Thus, it is difficult to distinguish between the effects of aging, cohorts, and period when either a cross-sectional or longitudinal design is employed.

To address these gaps, this study describes changes in $\mathrm{PA}$ and SB among Chinese children and adolescents using longitudinal data covering a period of 8 years. We explicitly assessed the differences in PA within individuals over time (age effect) and population-wide differences in PA overtime (period effect), and implicitly assessed the differences in the experienced period effect across individuals of varying ages (cohort effect). We performed this analysis while adjusting for social, economic, and environmental factors over time to determine how these factors contribute to changes in PA levels in Chinese children.

\section{Methods \\ Data}

The China Health and Nutrition Survey (CHNS) is a representative, prospective household-based study that includes multiple ages and cohorts across nine rounds of surveys between 1989 and 2011 in nine diverse provinces (Guangxi, Guizhou, Heilongjiang, Henan, Hubei, Hunan, Jiangsu, Liaoning, and Shandong) and three large cities (Beijing, Shanghai, and Chongqing were added in 2011) [22]. A multistage, stratified sampling design was used to ensure that the CHNS provided representation of rural, urban, and suburban areas that varied substantially by geography, economic development, public resources, and health indicators [22].

We believe this is the only large-scale, longitudinal study of its kind in China. Details of the study design have been described elsewhere [23].

Information on travel and active leisure activities was not available until 1997, while information on sedentary leisure activities was not collected until 2004 (e.g., watching TV). As a result, we focused on childhood PA using four rounds of comparable survey data collected in 2004, 2006, 2009, and 2011. Additionally, observations were excluded from the analysis if a participant was currently disabled at the time of data collection. We focused our analyses on children, defined here as between 6 to 17 (less than 18) years old.

Our final analysis included 3528 participants, with an average of 1.5 observations collected per subject (5864 observations). We excluded participants with only one observation. The data are reflective of an unbalanced panel with some individuals being absent in certain years and returning in future years as the sample was replenished over time. However, relative to previous rounds of data collection, participant retention was between $80 \%$ and $88 \%$ across all surveys after 2004 [22, 23]. 


\section{Outcomes}

The outcomes of interest were the metabolic equivalent of task (MET) hours per week (denoted as MET-hrs/wk). MET is defined as the ratio of a person's working metabolic rate relative to his or her resting (basal) metabolic rate [24]. Therefore, the MET-hrs/week measurement accounts for both the average intensity of each activity (or subactivity) and the time spent in each activity.

Due to our interest in measuring the changes in PA and SB across time periods, the primary outcome used here was limited to active MET-hrs/wk. from domestic, travel, leisure, work, and in-school activities, while sedentary MET-hrs/wk. were calculated from watching TV, videos or movies, board games, online-surfing or gaming, reading, playing chess or with dolls, and studying. We defined this as "PA" and "SB" in the results. First, each reported activity was assigned a MET value using the Compendium of Energy Expenditures for Youth for children. Average MET values were used for activity categories such as ball sports or other sports. The MET value for each activity was then multiplied by the total time spent per week (hrs/wk) in the activity, resulting in the METhrs/ wk. measurement. Details on how these values were calculated are described elsewhere $[25,26]$.

\section{Covariates}

\section{Age and gender}

Age in the baseline survey was used to define age groups of $6-8 y, 9-11 y, 12-14 y$, and $15-17 y$ for analysis which represented children and adolescents from junior primary school, senior primary school, middle school, and high school. Gender was also considered as a main covariate associated with $\mathrm{PA}$ and $\mathrm{SB}$.

\section{Urbanisation}

We calculated a multi-component urbanicity index from community survey data $[27,28]$, which reflected community infrastructure, population size and density, and economic and environmental characteristics. The scale illustrated misclassification using the traditional urbanrural dichotomy, and was able to detect differences in urbanicity, both between communities and across time. Furthermore, using a continuous measure of urbanicity allowed for better illustrations of the relationships between urbanicity and health. An increase in the value of the index over time represents greater urbanization.

\section{Asset ownership}

We were interested in the ownership of certain technologies or equipment related to PA and therefore included measurements of bicycle ownership, vehicle ownership, TV and computer ownership, and the number of computers at a household level.

\section{Individual and family factors}

We included individual level measures that may be associated with PA levels, which were weight status (normal weight vs. overweight/obese, which were defined using BMI cut-off points [29] for Chinese children and adolescents of 6-17 years old). We also accounted for household income (we generated ranked data by the quintiles in each wave) and household size (number of persons reported to be living in the household at the time of the survey) as potentially influential factors of PA.

\section{Statistical analysis}

Due to potential differences related to gender, we reported the PA and SB outcomes (active MET-hrs/wk. and sedentary MET-hrs/wk) and explanatory variables (age, percentage of males, being overweight/obese) as mean \pm SD or percentage stratified by gender for each survey year.

As the CHNS is a longitudinal study, attrition, migration, modifications to the sampling methods (including loss to follow-up and replenishment) over time, and other factors may result in cohort membership varying over time. In order to limit potential bias as a result of possible sample selection issues, we used a Heckmantwo-step approach [30] to calculate the "inverse Mill's ratio" for inclusion as a time-varying variable in all subsequent models described. The "inverse Mill's ratio" is the inverse of the predicted probability that an individual was included in each survey given his/her province, community urbanicity, and their interaction.

A series of longitudinal mixed effects models with fixed and random individual-level effects and random slopes was used to explicitly assess differences in PA within individuals over time (age effect) and populationwide differences in PA overtime (period effect). Finally, we implicitly assessed the differences in the experienced period effect across individuals of varying ages (cohort effect). Mixed effects models were chosen because they can accommodate imbalanced data and continuous covariates, and because the hierarchical nature of the models overcomes the identifiability problem by not assuming that age-period-cohort (APC) effects are linear and additive at the same level of analysis [31, 32].

We employed four models. Model 1 considers fundamental factors of age, indicator variables representing survey year, age interaction with survey year and gender. The year coefficients describe the period effect and interaction terms describe how period effects may differ by age (cohort effect). Model 2 included the inverse Mill's Ratio and community urbanicity index into model 1. We intended to estimate how these factors explain some of the period effects. Model 3 included individual and family level covariates (weight status and household size for example), asset ownership covariates (bicycle 
ownership and TV/computer ownership for example) into model 1. In addition to providing information about how these variables related to physical activity, comparing this model with the basic model provides information as to whether these individual factors can explain some of the period effects. Model 4 includes the full set of variables. All models were conducted using Stata's XTMIXED program [33]. Results were considered significant if $P<0.05$.

\section{Results}

The total sample sizes of children in the study were 1692 , 1357, 1198, and 1617 in 2004, 2006, 2009, and 2011 for each year. Cross-sectional analysis across survey years showed that the mean age of the included sample remained stable around from $12.5 \pm 3.3$ to $11.3 \pm 3.3$ years (Table 1 ). PA levels among Chinese children slightly decreased in MET hours across the 8-year period, from $50.0 \pm 63.2$ MET-hrs/wk. in 2004 to $47.1 \pm 54.9$ MET-hrs/wk. in 2011. The trends were similar for boys and girls. In contrast, SB among Chinese children increased in those years, from $31.8 \pm 22.0$ MET-hrs/wk. in 2004 to $37.6 \pm 22.2$ MET-hrs/ wk. in 2011. The percentage of SB from total PA increased from $38.5 \%$ to $44.4 \%$, from $35.8 \%$ to $41.6 \%$, and from $40.1 \%$ to $45.7 \%$ among all boys and girls, for the different study years respectively. Meanwhile, the prevalence of being overweight and obese in Chinese children increased between 2004 and 2011 from $11.3 \%$ to $19.8 \%$, from $11.7 \%$ to $23.4 \%$, from $10.8 \%$ to $15.9 \%$ among all boys and girls, for the different study years respectively.

\section{Physical activity (PA)}

In Fig. 1, PA levels in boys from the four baseline age groups are shown according to the mean age of the group by each survey year. Each point represents a survey year, and the lines span the 8-year period over which the PA data was collected. Younger boys had lower initial PA, and with age, PA levels changed differently across baseline age groups. The relationship between age and mean PA is non-linear. Meanwhile, the vertical dotted line illustrates the difference in estimated PA level for 13-year old boys in 2004 (65 MET-hr./wk) compared to 13-year old boys in 2006 (60 MET-hr./wk), and in 2011 (50 MET-hr./wk). This is deemed the cohort effect. PA levels of girls in the four baseline age groups are also shown according to the mean age of the group by each survey year in Fig. 2. A similar trend was observed for girls with respect to reduced PA levels while the cohort effect was also observed.

The non-linear age-related patterns of reductions in PA were confirmed by the longitudinal analysis. An age effect was not observed by adding age-square into the models.

Table 1 Cross-sectional univariate descriptives of the China Health and Nutrition Survey across survey years, mean \pm SD

\begin{tabular}{|c|c|c|c|c|}
\hline & \multicolumn{4}{|l|}{ Survey years } \\
\hline & 2004 & 2006 & 2009 & 2011 \\
\hline All Participants(N) & 1692 & 1357 & 1198 & 1617 \\
\hline Age & $12.45 \pm 3.33$ & $11.91 \pm 3.36$ & $11.59 \pm 3.19$ & $11.33 \pm 3.33$ \\
\hline Male(\%) & 53.0 & 53.4 & 55.8 & 51.1 \\
\hline Overweight/Obese(\%) & 11.3 & 12.0 & 14.5 & 19.8 \\
\hline PA MET-hours/week & $50.00 \pm 63.15$ & $47.56 \pm 61.78$ & $46.51 \pm 56.73$ & $47.14 \pm 54.86$ \\
\hline SB MET-hours/week & $31.76 \pm 22.00$ & $34.85 \pm 21.00$ & $35.54 \pm 21.83$ & $37.62 \pm 22.24$ \\
\hline$\%$ SB in total PA & 38.5 & 42.3 & 43.3 & 44.4 \\
\hline Male(N) & 896 & 725 & 668 & 826 \\
\hline Age & $12.57 \pm 3.41$ & $11.86 \pm 3.40$ & $11.57 \pm 3.23$ & $11.29 \pm 3.31$ \\
\hline Overweight/Obese(\%) & 11.7 & 13.3 & 16.4 & 23.4 \\
\hline PA MET-hours/week & $57.15 \pm 68.65$ & $54.30 \pm 68.07$ & $52.66 \pm 61.07$ & $53.56 \pm 60.52$ \\
\hline SB MET-hours/week & $31.89 \pm 22.89$ & $36.32 \pm 23.05$ & $35.06 \pm 21.62$ & $38.10 \pm 24.48$ \\
\hline$\% \mathrm{SB}$ in total $\mathrm{PA}$ & 35.8 & 40.1 & 40.0 & 41.6 \\
\hline Female(N) & 796 & 632 & 530 & 791 \\
\hline Age & $12.30 \pm 3.22$ & $11.97 \pm 3.31$ & $11.62 \pm 3.15$ & $11.38 \pm 3.36$ \\
\hline Overweight/Obese $(\%)^{a}$ & 10.8 & 10.5 & 12.0 & 15.9 \\
\hline PA MET-hours/week & $47.24 \pm 57.27$ & $45.46 \pm 54.98$ & $43.66 \pm 51.34$ & $44.13 \pm 48.49$ \\
\hline SB MET-hours/week & $31.61 \pm 20.97$ & $33.17 \pm 18.24$ & $36.16 \pm 22.10$ & $37.11 \pm 19.62$ \\
\hline$\%$ SB in total PA & 40.1 & 42.2 & 45.3 & 45.7 \\
\hline
\end{tabular}

a Using the criteria of overweight and obesity for Chinese children

$P A$ physical activity, SB sedentary behaviour 


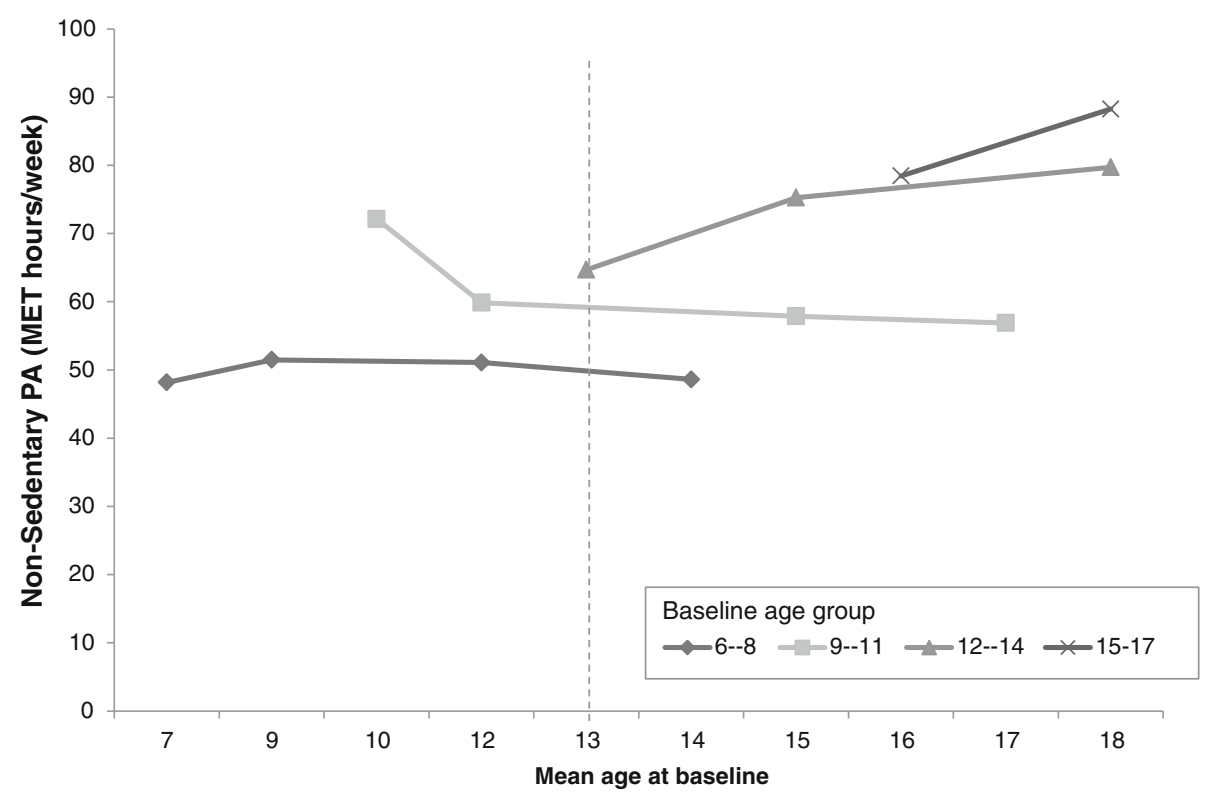

Fig. 1 Age trends in mean physical activity among boys in the CHNS by baseline age groups (MET-hrs/wk). Notes: Points represent PA level of boys at the mean age for the age group in each survey year $(2004,2006,2009,2011)$. The vertical dotted line illustrates the difference in estimated PA level for a 13-year old boy in 2004 vs 13-year old boy in 2006 vs a 13-year old boy in 2009 (cohort effect).

When adding age-square into the models, we found the coefficients for age and age-square contrasted with each other. Moreover, the age trend was not the same when different baseline age groups were examined (Fig. 1). Controlling for age, we found that being female was negatively associated with PA. The model results showed that period effects were positively associated with PA in 2011, when controlling for age and gender (Table 2, model 1).
However, the period effect was extinguished after we adjusted for other covariates. The interaction between survey year and age $(p<0.05)$ was observed in 2011 (Table 2 , model 1 ). In model 2 , higher community urbanicity was found as a negative predictor of PA (Table 2, model 2). In model 3, we found that the addition of one extra computer per family resulted in a change in PA by -4.80 (-9.03,-0.57) MET-hr./wk. After adjusting for potential

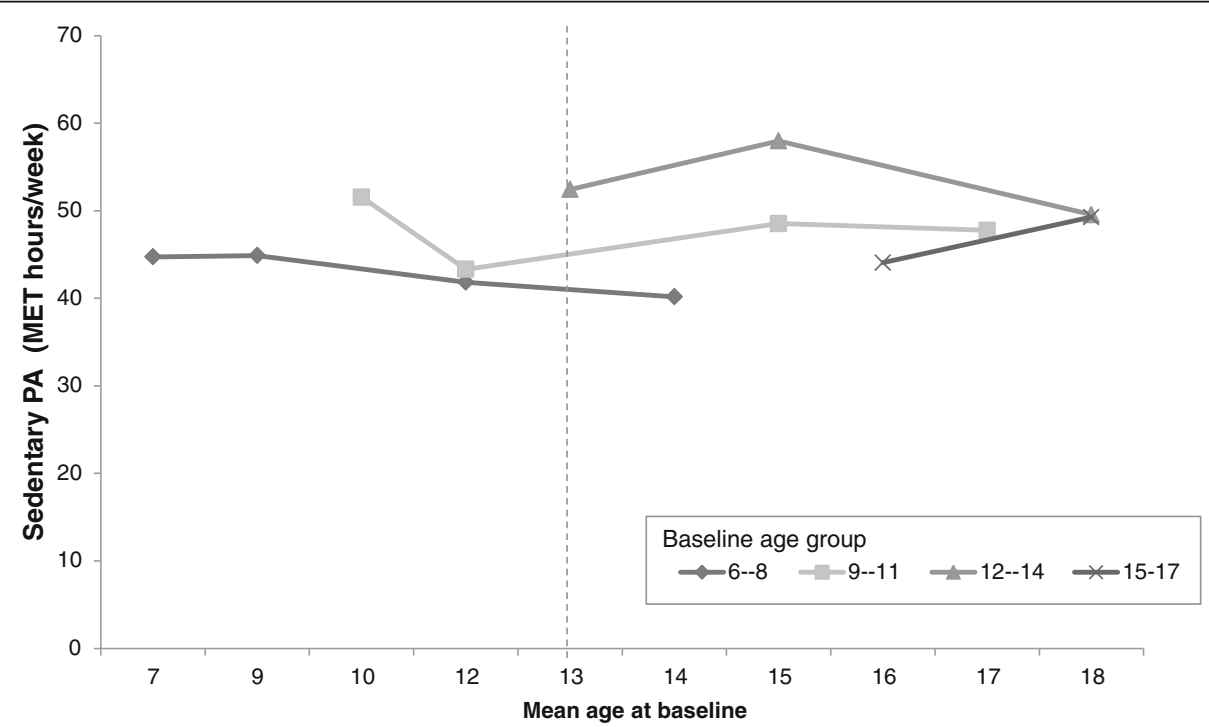

Fig. 2 Age trends in mean physical activity among girls in the CHNS by baseline age groups (MET-hrs/wk). Notes: Points represent PA level of female children at the mean age for the age group in each survey year $(2004,2006,2009,2011)$. The vertical dotted line illustrates the difference in estimated PA level for a 13-year old girl in 2004 vs 13-year old girl in 2006 vs a 13-year old girl in 2009 (cohort effect) 
Table 2 Mixed effects estimates on physical activity (PA) levels (MET-hrs/wk) among Chinese children, coefficient (95\% Cl)

\begin{tabular}{|c|c|c|c|c|}
\hline$\overline{P A}$ & Model 1 & Model 2 & Model 3 & Model 4 \\
\hline Age & $6.12(5.28,6.96)$ & $6.21(5.36,7.07)$ & $5.6(4.72,6.49)$ & $5.75(4.84,6.65)$ \\
\hline \multicolumn{5}{|l|}{ Survey year (ref: 2004) } \\
\hline 2006 & $0.40(-13.74,14.55)$ & $-1.05(-15.42,13.31)$ & $-3.31(-18.61,11.99)$ & $-5.18(-20.7,10.34)$ \\
\hline 2009 & $12.98(-2.78,28.74)$ & $12.64(-3.25,28.54)$ & $10.15(-6.39,26.69)$ & $9.97(-6.70,26.64)$ \\
\hline 2011 & $-23.6(-9.14,-38.07)$ & $-17.73(-1.77,-33.69)$ & $-20.2(-5.28,-35.13)$ & $14.56(-1.95,31.06)$ \\
\hline $2006^{*}$ Age & $-0.10(-1.26,1.07)$ & $-0.08(-1.25,1.1)$ & $0.41(-0.84,1.66)$ & $0.40(-0.86,1.66)$ \\
\hline $2009^{*}$ Age & $-1.04(-2.37,0.29)$ & $-1.01(-2.35,0.34)$ & $-0.60(-1.98,0.79)$ & $-0.65(-2.05,0.76)$ \\
\hline $2011^{*}$ Age & $-1.91(-3.15,-0.68)$ & $-1.20(-2.55,0.15)$ & $-1.31(-2.57,-0.05)$ & $-0.96(-2.04,-0.73)$ \\
\hline Gender & $-6.38(-9.85,-2.9)$ & $-5.86(-9.52,-2.2)$ & $-6.24(-9.73,-2.75)$ & $-5.74(-9.42,-2.06)$ \\
\hline Inverse Mill's Ratio & & $3.99(-1.51,9.48)$ & & $5.80(0.06,11.53)$ \\
\hline Community Urbanicity & & $-0.15(-0.24,-0.05)$ & & $-0.07(-0.18,-0.03)$ \\
\hline Bicycle ownership & & & $2.07(-1.24,5.37)$ & $2.34(-1.12,5.79)$ \\
\hline Vehicle ownership & & & $0.71(-2.58,4.01)$ & $0.06(-3.42,3.53)$ \\
\hline No of Computers & & & $-4.80(-9.03,-0.57)$ & $-4.74(-9.43,-0.05)$ \\
\hline No of Household members & & & $0.71(-0.57,1.99)$ & $0.41(-0.95,1.77)$ \\
\hline Overweight/Obese & & & $0.61(-3.91,5.14)$ & $2.88(-1.97,7.72)$ \\
\hline Household income & & & $0.60(-1.68,2.89)$ & $0.65(-1.79,3.08)$ \\
\hline Number of observations & 5697 & 5319 & 4532 & 4177 \\
\hline Number of individuals & 3528 & 3162 & 3022 & 2676 \\
\hline
\end{tabular}

Notes: Data from CHNS 2004-2011; bold values denote statistical significance at $p<0.05$

confounding effects of age, gender, the interaction of 2011 and age, and community urbanicity, the number of computers per family was identified as a negatively associated factor of PA (Table 2, model 4).

In comparing the coefficients among models, we demonstrated that the number of computers per family was significantly higher and accounted for most of the period effects besides age and gender.

\section{Sedentary behaviour (SB)}

SB levels of boys in the four baseline age groups are shown according to the mean age of the group by each survey year in Fig. 3. Each point represents a survey year and the lines span the 8-year period over which the PA data was collected. Younger boys had lower initial SB, and with age, PA levels increased across all baseline age groups. However, the relationship between age and mean $\mathrm{SB}$ is non-linear. Meanwhile, the vertical dotted line illustrates the difference in estimated PA level for 13-year old boys in 2004 (33 MET-hr./wk) compared to 13-year old boys in 2006 (36 MET-hr./wk), and in 2011 (37 MET-hr./wk). This is evidence of the cohort effect.

The SB levels of girls in the four baseline age groups are also shown according to the mean age of the group by each survey year in Fig. 4. A similar trend was observed to the boys in that the level of SB increased by cohort year.
The non-linear age-related patterns of reductions in SB were identified by the longitudinal analysis. Higher age was positively associated with SB but gender was not associated with SB. The model results showed that time period was positively associated with PA in 2009, when controlling for age and gender (Table 3, model 1). The interaction between survey year and age $(p<0.05)$ was observed in 2006 (Table 3, model 1). However, the effect disappeared in model 4 . In model 2 , higher community urbanicity was found as a positive predictor of $\mathrm{SB}$ (Table 3, model 2). In model 3, we found that the number of computers owned and higher household incomes were positively associated with SB. In contrast, larger household size was a negative predictor of SB. After adjusting for potential confounding the effects of community urbanicity, number of computers per family, household size, and household income were identified as positive predictors of SB notwithstanding age (Table 3, model 4). Potential secular trends of SB was observed for both boys and girls (Additional file 1: Figure S1 and Additional file 2: Figure S2).

\section{Discussion}

This study included Chinese children aged 6-17 years old between 2004 and 2011, and attempted to elucidate the epidemiological importance of age, period, and cohort changes in primary sources of both PA and SB, using a reliable, validated PA questionnaire [34-37]. 


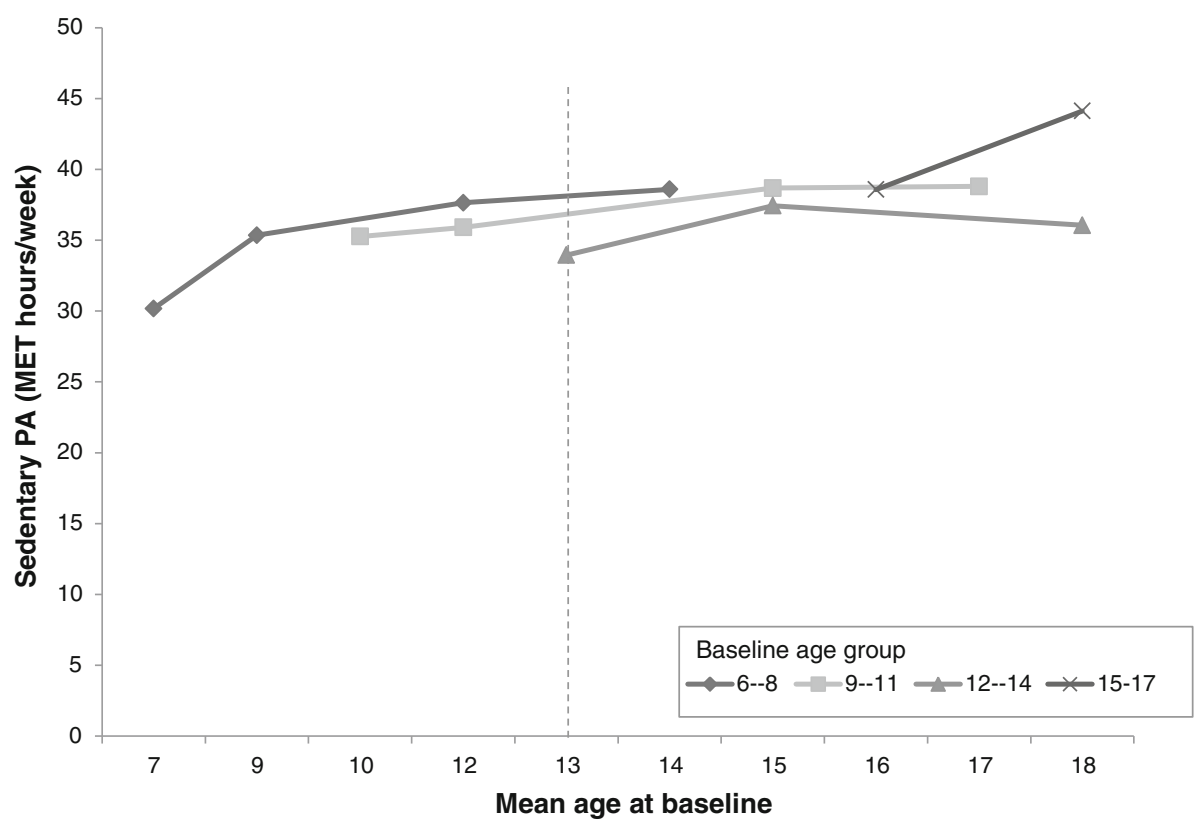

Fig. 3 Age trends in mean Sedentary behaviour among boys in the CHNS by baseline age groups (MET-hrs/wk). Notes: Points represent Sedentary behaviour level of boys at the mean age for the age group in each survey year $(2004,2006,2009,2011)$. The vertical dotted line illustrates the difference in estimated PA level for a 13-year old boy in 2004 vs 13-year old boy in 2006 vs a 13-year old boy in 2009 (cohort effect)

Throughout this 8-year period, we found that PA levels decreased slightly but that SB moderately increased for both boys and girls. The proportion of SB from total PA was more pronounced in girls than boys and increased in both gender groups across time. Being older was associated with SB while being female was negatively associated with PA but not with SB. For PA, higher community urbanicity, more computers per family, and small period effects were found to be negative predictors of SB. We found that higher community

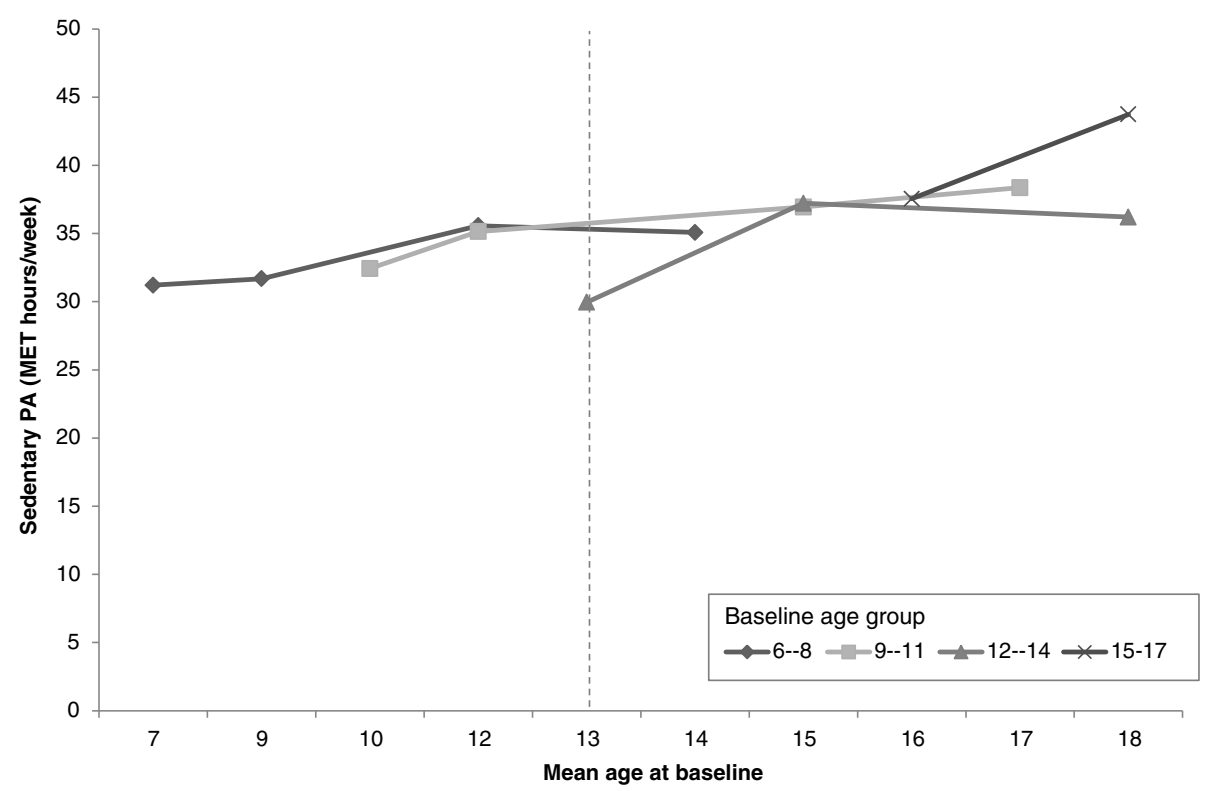

Fig. 4 Age trends in mean Sedentary behaviour among girls in the CHNS by baseline age groups (MET-hrs/wk). Notes: Points represent Sedentary behaviour level of female children at the mean age for the age group in each survey year $(2004,2006,2009,2011)$. The vertical dotted line illustrates the difference in estimated PA level for a 13-year old girl in 2004 vs 13-year old girl in 2006 vs a 13-year old girl in 2009 (cohort effect) 
Table 3 Mixed effects estimates on sedentary behavior (SB) levels (MET-hrs/wk) among Chinese children, coefficient (95\% Cl)

\begin{tabular}{|c|c|c|c|c|}
\hline SB & Model 1 & Model 2 & Model 3 & Model 4 \\
\hline Age & $0.64(0.34,0.95)$ & $0.53(0.22,0.84)$ & $0.57(0.25,0.89)$ & $0.49(0.17,0.81)$ \\
\hline \multicolumn{5}{|l|}{ Survey year (ref: 2004) } \\
\hline 2006 & $-1.99(-7.56,3.57)$ & $-0.82(-6.45,4.8)$ & $-1.14(-7,4.72)$ & $-0.28(-6.22,5.66)$ \\
\hline 2009 & $7.41(1.43,13.38)$ & $6.8(0.82,12.79)$ & $6.54(0.39,12.69)$ & $5.86(-0.33,12.04)$ \\
\hline 2011 & $2.97(-2.4,8.34)$ & $0.86(-5.04,6.75)$ & $2.61(-2.85,8.08)$ & $1.24(-4.79,7.27)$ \\
\hline $2006^{*}$ Age & $0.45(0.00,0.89)$ & $0.45(0.00,0.9)$ & $0.37(-0.10,0.84)$ & $0.39(-0.09,0.86)$ \\
\hline $2009^{*}$ Age & $-0.26(-0.75,0.23)$ & $-0.19(-0.69,0.3)$ & $-0.25(-0.75,0.26)$ & $-0.15(-0.66,0.36)$ \\
\hline $2011^{*}$ Age & $0.30(-0.14,0.74)$ & $0.23(-0.26,0.71)$ & $0.16(-0.29,0.61)$ & $0.12(-0.37,0.61)$ \\
\hline Gender & $-0.95(-2.12,0.22)$ & $-0.89(-2.1,0.32)$ & $-0.69(-1.86,0.49)$ & $-0.54(-1.77,0.69)$ \\
\hline Inverse Mill's Ratio & & $-3.64(-5.61,-1.68)$ & & $-3.21(-5.25,-1.17)$ \\
\hline Community Urbanicity & & $0.11(0.08,0.14)$ & & $0.06(0.03,0.10)$ \\
\hline Bicycle ownership & & & $0.50(-0.68,1.68)$ & $0.37(-0.86,1.6)$ \\
\hline Vehicle ownership & & & $-0.87(-2.04,0.31)$ & $0.06(-1.18,1.3)$ \\
\hline No of Computers & & & $3.43(2.28,4.58)$ & $2.41(1.12,3.71)$ \\
\hline No of Household members & & & $-1.12(-1.56,-0.67)$ & $-0.89(-1.36,-0.42)$ \\
\hline Overweight/Obese & & & $1.32(0.00,2.64)$ & $1.5(-0.14,3.13)$ \\
\hline Household income & & & $1.45(0.63,2.28)$ & $0.93(0.04,1.81)$ \\
\hline Number of observations & 5697 & 5319 & 4532 & 4177 \\
\hline Number of individuals & 3528 & 3162 & 3022 & 2676 \\
\hline
\end{tabular}

Notes: Data from CHNS 2004-2011; bold values denote statistical significance at $p<0.05$

urbanization, increased computer appliance ownership, and higher household income were positively associated with SB. Surprisingly, larger household size was found to be the only factor that was negatively associated with SB.

The CHNS was designed to provide representation of rural, urban, and suburban areas that vary substantially in geography, economic development, public resources, and health indicators. It is the only large-scale, longitudinal study of its kind in China [23]. It mainly focuses on household- and individual-level socio-demographic factors, diet, physical activity, health and behavioural changes within the context of community-level urbanicity, and social and economic change [22].

Accumulating evidence shows that higher levels of SB are associated with a higher risk of obesity, less healthy diet, and lower cardiopulmonary fitness in children and adolescents [38-40]. It is important to independently assess PA levels separately from SB. The PA levels of Chinese children have not changed significantly from 2004 to 2011. The government of China established a regulation to promote one hour of exercise to school students since 2007 [41]. This was done to prevent a dramatic decline in PA since most children and adolescents are school students.

Our study found that PA levels only slightly declined but that SB increased by $18.5 \%$ from 2004 to 2011 . Several other studies corroborate that there is an increase in SB [1, 42, 43]. A previous study found that Chinese students are under extreme pressure to perform well in academic settings [44]. They spend more time on homework or other sedentary learning activities (e.g., drawing and playing chess). In another study, the cohorts experienced rapid environmental change at different ages with changes related to China's dramatic economic, demographic, and social transformation. These changes included modernization of the Chinese food system, continued use and greater dissemination of modern technology in manufacturing, transportation and leisure, environmental and social systems, and changes to health system and educational systems [45, 46]. Moreover, technological advances in travel and leisure time have reduced the physical demands in many aspects. Those changes might result in an improved level of urbanisation, varying the way people live, while ultimately increasing SB levels across time.

The gross domestic product (GDP) of China increased 3.3-fold from 2004 to 2011 and the urbanization rate raised increased by nearly $10 \%$ in 8 years (from $41.8 \%$ in 2004 to $51.3 \%$ in 2011) [47]. Our data showed that the greater the level of community urbanization, the more likely this would be associated with higher levels of SB, and lower levels of PA. Increased community urbanization was associated with an increase in less laborious transportation, easier access to TV and computer appliances, and augmented academic pressure for Chinese children, which were all related to increased levels of SB and decreased PA, respectively. 
The proportion of Chinese families who own colour televisions, have access to cable networks and computers has all also increased dramatically, as has screen time [48-50]. We initially included TV and computer ownership as a potential covariate into the models, however more than $90 \%$ of Chinese families owned a TV between 2004 and 2011. Thus, we did not believe it was not necessary to include TV ownership. Interestingly, we found that increased computer ownership was associated with the decline of PA in children, which conflicts with some other studies assessing the role of computer use $[1,21,40]$. On the contrary, increased level of computer ownership was associated with an increase of SB in Chinese children.

We found that being overweight or obese was marginally associated with higher SB. This result was consistent with some other studies $[1,3]$. Weight status was related to other aforementioned factors described previously. Higher household income was associated with a lower level of SB, which was consistent with another study [51].

To our surprise, large household size was found to be the only factor that was negatively associated with SB in Chinese children. The reason for this might be due to family or siblings encouraging higher levels of physical activities [52]. Many Chinese families have only been able to have one child as a result of the 'one-child' policy since 1979 (which was only recently altered) [53]. Consequently, it is possible that single children have been overprotected by their parents in all aspects when compared to families with two children or more, and this may result in increased SB for only children. Besides, children with siblings may have someone to play with and influence each other [54]. We further analyzed the PA levels among single children and children with siblings. The results showed that PA levels were $46.2 \pm 58.4$ MET-hr./wk., $53.9 \pm 76.8$ MET-hr./wk., and $66.8 \pm 92.9$ MET-hr./wk. for a single child, children with one sibling, and children with more than one sibling, respectively. Moreover, the SB levels were $36.6 \pm 21.9$ MET-hr./wk., $32.9 \pm 22.3$ MET-hr./wk., and $28.0 \pm 18.8$ MET-hr./wk. for a single child, children with one sibling, and children with more than one sibling, respectively. From January 1, 2016, all Chinese couples are allowed to have two children. This marked the end of China's 'one-child' policy, which had restricted the majority of Chinese families to have only one child for more than 35 years. Previously, we analyzed the PA level of adult females using CHNS data from 1991 to 2011 and we also found that bigger household size was positively associated with work and domestic PA [55]. The change in policy might positively influence children's PA levels in a positive manner and might promote good health for both children and mothers.

Girls had lower PA levels than boys but SB levels were no different. However, the SB level of boys and girls was similar and the increasing trends were also similar for both genders. A previous study reported that SB was easier for girls to control but this was not the case for boys [56]. The study indicated that if girls and boys had the same SB levels, more attention should be focused on boys since overweight and obese male adults are more likely to suffer poor cardiovascular health compared with their female counterparts.

An important strength of this study is that this is the largest longitudinal analysis of physical activity to be conducted in a diverse sample of Chinese children. We separately assessed PA and SB changes among Chinese children while differentiating between effects of age, time period, and birth cohort. This allowed us to robustly determine how physical activity is influenced by biological, behavioral, economic, and environmental factors over time. To our knowledge, we believe this is the first study to assess these phenomena and reveal several important predictors for both PA and SB. The findings of our study may shed light on design of urbanization for policy-makers, while endorsing government-initiated health promotion and recommendation of physical activity for school age children.

Nonetheless, there are several limitations to this study. First, recall and social desirability biases exist in selfreported PA data, although it is not clear whether PA is overestimated due to social desirability bias in some developed countries [57]. Second, the collection methods of PA data in children and adolescents varied slightly by age groups, with assistance from caregivers for children $<10$ years. While different data collection methods were used to improve accuracy of PA, [58] comparisons with CHNS PA data in children based on parent-assisted selfreports and self-reports has not yet been conducted. Thirdly, we did not assess moderate to vigorous physical activity in our study, which may be more significant for public health issues. We separately analyzed physical activity as the physical activity and SB in our study. It is likely that moderate to vigorous physical activity MET hours contributed to most of the total PA MET hours.

Poor PA, especially physical activity, contributes to between 12 and 19\% of the five major NCDs in China and the direct and indirect costs of physical inactivity in China has been estimated at 6.7 billion USD (in 2007 dollars) [59]. This cost is expected to grow as PA levels continue to decrease, given the potential age effect and secular trends observed in this study, as well as the much higher SB levels and lower PA levels of the younger cohorts.

\section{Conclusion}

In summary, this study followed a large cohort of children over a significant period of their childhood. As China underwent dramatic economic transition, we 
observed potential age and secular trends in PA levels. Community urbanisation, computer ownership, and household size were related to PA levels. These findings are highly relevant for health policy and preventative health measures in China and other countries that are now facing similar public health challenges.

\section{Additional files}

Additional file 1: Figure S1. Secular trends in SB level among boys in CHNS by baseline age groups. Notes: Bars represent difference from baseline (2004) Sedentary Activity, estimated from longitudinal models, stratified by baseline age groups. (PDF $285 \mathrm{~kb}$ )

Additional file 2: Figure S2. Secular trends in SB level among girls in CHNS by baseline age groups. Notes: Bars represent difference from baseline (2004) Sedentary Activity, estimated from longitudinal models, stratified by baseline age groups. (PDF $248 \mathrm{~kb}$ )

\section{Abbreviations}

PA: Physical activity; CHNS: China Health and Nutrition Survey;

MET: Metabolic equivalent of task; MET-hr./wk.: MET hours per week; DALYs: Disability-adjusted life-years; NCDs: Non-communicable diseases; APC: Age-period-cohort

\section{Acknowledgements}

We acknowledged Dr. Shufa Du from the University of North Carolina at Chapel Hill for helping us with data access. And we acknowledge the grants from Shanghai Rising-Star Program (number: 15YF1411100), Shanghai Municipal Bureau of Health (number: 20134053), the Natural Science Foundation of Shanghai (No. 14ZR1449500), the National Natural Science Foundation of China (No.81401855 and No. 81602851) and the Fourth 3-year Public Health Action Plan in Shanghai, China (No. GWIV-27.1).

\section{Funding}

This work was supported by grants from Shanghai Municipal Bureau of Health (number: 20,134,053), Shanghai Rising-Star Program (number: 15YF1411100), the Natural Science Foundation of Shanghai (No. 14ZR1449500) and the National Natural Science Foundation of China (No.81401855 and No. 81602851). The funders had no role in study design, data collection and analysis, decision to publish, or preparation of the article.

\section{Availability of data and materials}

The datasets generated and/or analysed during the current study are available in the following website: http://www.cpc.unc.edu/projects/china/.

\section{Authors' contributions}

$J Z, X W, M S, Y Z$ and $X J$ conceived and designed the experiments; XH, SZ, HW analyzed the data; JZ, XW, MS, YZ and XJ wrote the paper. All authors read and approved the final manuscript.

\section{Competing interests}

The authors declare that they have no competing interests.

\section{Consent for publication}

Not applicable.

\section{Ethics approval and consent to participate}

The CHNS was approved by the institutional review committees of the University of North Carolina at Chapel Hill, the National Institute of Nutrition and Food Safety and China Center for Disease Control and Prevention. Written informed consent was obtained from the human subjects. The manuscript did not contain any individual person's data in any form.

\section{Publisher's Note}

Springer Nature remains neutral with regard to jurisdictional claims in published maps and institutional affiliations.

\section{Author details}

Gu Mei Community Service Center, 668 Longming Road, Shanghai 200010, People's Republic of China. ${ }^{2}$ Department of Neurology, the Affiliated Hospital of North China University of Science and Technology, No.73 south construction road, Tangshan, Hebei, People's Republic of China. ${ }^{3}$ Department of Nutrition Hygiene, Division of Health Risk Factor Monitoring and Control, Shanghai Municipal Center for Disease Control and Prevention, 1380 West Zhongshan Road, Changning District, Shanghai 200336, People's Republic of China. ${ }^{4}$ Department of Preventative Ophthalmology, Shanghai Eye Disease Prevention and Treatment Center, Shanghai Eye Hospital, Shanghai 200040 People's Republic of China. ${ }^{5}$ Department of Epidemiology, School of Public Health, Nanjing Medical University, 101 Longmian Ave, Jiangning District, Nanjing 211166, Jiangsu, People's Republic of China. 'Department of Intensive Care Unit, Shanghai Provincial Crops Hospital, Chinese People's Armed Police Forces, 831 Hongxu Road, Shanghai 201103, People's Republic of China. ${ }^{7}$ Key Laboratory of Biomedical Information, Engineering, Ministry of Education, Institute of Mitochondrial Biology and Medicine, Xi'an Jiaotong University School of Life Science and Technology, Xi'an 710049, People's Republic of China.

Received: 13 July 2016 Accepted: 1 April 2017

Published online: 24 April 2017

References

1. Duan J, Hu H, Wang G, Arao T. Study on current levels of physical activity and sedentary behavior among middle school students in Beijing, China. PloS one. 2015;10(7):e0133544.

2. Ng M, Fleming T, Robinson M, et al. Global, regional, and national prevalence of overweight and obesity in children and adults during 19802013: a systematic analysis for the Global burden of disease study 2013. Lancet. 2014:384(9945):766-81.

3. Sigmund E, Sigmundova D, Badura P, Kalman M, Hamrik Z, Pavelka J. Temporal trends in overweight and obesity, physical activity and screen time among Czech adolescents from 2002 to 2014: a National Health Behaviour in school-aged children study. Int J Environ Res Public Health. 2015;12(9):11848-68

4. Mokabane NN, Mashao MM, van Staden M, Potgieter M, Potgieter A. Low levels of physical activity in female adolescents cause overweight and obesity: are our schools failing our children? South African medical journal = Suid-Afrikaanse tydskrif vir geneeskunde. 2014;104(10):665-7.

5. Johnson RK, Russ J, Goran MI. Physical activity related energy expenditure in children by doubly labeled water as compared with the Caltrac accelerometer. International journal of obesity and related metabolic disorders : journal of the International Association for the Study of Obesity. 1998;22(11):1046-52.

6. Steinberger J, Daniels SR, Eckel RH, et al. Progress and challenges in metabolic syndrome in children and adolescents: a scientific statement from the American Heart Association atherosclerosis, hypertension, and obesity in the young Committee of the Council on cardiovascular disease in the young; Council on cardiovascular nursing; and Council on nutrition, physical activity, and metabolism. Circulation. 2009;119(4):628-47.

7. Lees C, Hopkins J. Effect of aerobic exercise on cognition, academic achievement, and psychosocial function in children: a systematic review of randomized control trials. Prev Chronic Dis. 2013;10:E174.

8. WH Organization. Global Recommendations on Physical Activity for Health. Geneva: WH Organization; 2010. Available at: http://www.who.int/ dietphysicalactivity/factsheet_recommendations/en/.

9. World Health Organization. Global Recommendations on Physical Activityfor Health, 2012, 2011: 5-17 year olds. Available at: http://www.who.int/ dietphysicalactivity/physical-activity-recommendations-5-17years.pdf\%3E. Accessed 12 Aug 2012.

10. Katzmarzyk PT. Physical activity, sedentary behavior, and health: paradigm paralysis or paradigm shift? Diabetes. 2010;59(11):2717-25.

11. Ekelund U, Brage S, Froberg K, et al. TV viewing and physical activity are independently associated with metabolic risk in children: the European youth heart study. PLoS Med. 2006;3(12):e488.

12. Danielsen YS, Juliusson PB, Nordhus IH, et al. The relationship between lifestyle and cardio-metabolic risk indicators in children: the importance of screen time. Acta Paediatr. 2011;100(2):253-9.

13. Hardy LL, Denney-Wilson E, Thrift AP, Okely AD, Baur LA. Screen time and metabolic risk factors among adolescents. Archives of pediatrics \& adolescent medicine. 2010;164(7):643-9. 
14. Kang HT, Lee HR, Shim JY, Shin YH, Park BJ, Lee YJ. Association between screen time and metabolic syndrome in children and adolescents in Korea: the 2005 Korean National Health and nutrition examination survey. Diabetes Res Clin Pract. 2010;89(1):72-8.

15. Nang EE, Salim A, Wu Y, Tai ES, Lee J, Van Dam RM. Television screen time, but not computer use and reading time, is associated with cardio-metabolic biomarkers in a multiethnic Asian population: a cross-sectional study. The international journal of behavioral nutrition and physical activity. 2013;10:70.

16. Chen $J L$, Wu Y. Cardiovascular risk factors in Chinese American children: associations between overweight, acculturation, and physical activity. Journal of pediatric health care : official publication of National Association of Pediatric Nurse Associates \& Practitioners. 2008;22(2):103-10.

17. Huang WY, Wong SH, Salmon J. Correlates of physical activity and screenbased behaviors in Chinese children. Journal of science and medicine in sport / Sports Medicine Australia. 2013;16(6):509-14.

18. Wang C, Chen P, Zhuang J. A national survey of physical activity and sedentary behavior of Chinese city children and youth using accelerometers. Res Q Exerc Sport. 2013;84(Suppl 2):S12-28.

19. Li L, Li K, Ushijima H. Moderate-vigorous physical activity and body fatness in Chinese urban school children. Pediatrics international : official journal of the Japan Pediatric Society. 2007:49(2):280-5.

20. Dearth-Wesley T, Gordon-Larsen P, Adair LS, Zhang B, Popkin BM. Longitudinal, cross-cohort comparison of physical activity patterns in Chinese mothers and children. The international journal of behavioral nutrition and physical activity. 2012;9:39.

21. Wong SH, Huang WY, He G. Longitudinal changes in objectively measured physical activity differ for weekdays and weekends among Chinese children in Hong Kong. BMC Public Health. 2015;15:1310.

22. Popkin BM, Du S, Zhai F, Zhang B. Cohort profile: the China health and nutrition survey-monitoring and understanding socio-economic and health change in China, 1989-2011. Int J Epidemiol. 2010;39(6):1435-40.

23. Zhang B, Zhai FY, Du SF, Popkin BM. The China health and nutrition survey, 1989-2011. Obesity reviews : an official journal of the International Association for the Study of Obesity. 2014;15(Suppl 1):2-7.

24. Sallis JF, Haskell WL, Wood PD, et al. Physical activity assessment methodology in the Five-City project. Am J Epidemiol. 1985:121(1):91-106.

25. Ng SW, Popkin BM. Time use and physical activity: a shift away from movement across the globe. Obes Rev. 2012;13(8):659-80.

26. Ng SW, Norton EC, Popkin BM. Why have physical activity levels declined among Chinese adults? Findings from the 1991-2006 China health and nutrition surveys. Soc Sci Med. 2009;68(7):1305-14.

27. Dahly DL, Adair LS. Quantifying the urban environment: a scale measure of urbanicity outperforms the urban-rural dichotomy. Soc Sci Med. 2007;64(7):1407-19.

28. Jones-Smith JC, Popkin BM. Understanding community context and adult health changes in China: development of an urbanicity scale. Soc Sci Med. 2010;71(8):1436-46.

29. Group of China Obesity Task Force. Body mass index reference norm for screening overweight and obesity in Chinese children and adolescents. Chinese J Epidemiol. 2004;25:97-102.

30. Heckman JJ. Sample selection bias as a specification error. Econometrica. 1979;47:153-61.

31. Yang $Y$, Lee LC. Dynamics and heterogeneity in the process of human frailty and aging: evidence from the U.S. older adult population. J Gerontol Ser B Psychol Sci Soc Sci. 2010;65B(2):246-55.

32. Yang $Y$, Lee LC. Sex and race disparities in health: cohort variations in life course patterns. Social Forces. 2009;87:2093-124.

33. StataCorp. Stata statistical software, release 11. College Station (TX): StataCorp LP; 2009.

34. Bassett Jr DR. International physical activity questionnaire: 12-country reliability and validity. Med Sci Sports Exerc. 2003;35(8):1396.

35. Bauman A, Bull F, Chey T, et al. The International Prevalence Study on Physical Activity: results from 20 countries. The international journal of behavioral nutrition and physical activity 2009; 6: 21

36. Craig $\mathrm{CL}$, Marshall $\mathrm{AL}$, Sjostrom $\mathrm{M}$, et al. International physical activity questionnaire: 12-country reliability and validity. Med Sci Sports Exerc. 2003;35(8):1381-95.

37. Guthold R, Ono T, Strong KL, Chatterji S, Morabia A. Worldwide variability in physical inactivity a 51-country survey. Am J Prev Med. 2008;34(6):486-94.

38. Rey-Lopez JP, Vicente-Rodriguez G, Biosca M, Moreno LA. Sedentary behaviour and obesity development in children and adolescents. Nutrition, metabolism, and cardiovascular diseases : NMCD. 2008;18(3):242-51.
39. Martinez-Gomez D, Ortega FB, Ruiz JR, et al. Excessive sedentary time and low cardiorespiratory fitness in European adolescents: the HELENA study. Arch Dis Child. 2011;96(3):240-6.

40. Hobbs M, Pearson N, Foster PJ, Biddle SJ. Sedentary behaviour and diet across the lifespan: an updated systematic review. Br J Sports Med. 2015; 49(18):1179-88

41. The Central People's Government of the People's Republic of China. http://www. gov.cn/gongbao/content/2007/content_663655.htm. Accessed 15 Feb 2016.

42. Li M, Dibley MJ, Sibbritt DW, Zhou X, Yan H. Physical activity and sedentary behavior in adolescents in Xi'an City, China. The Journal of adolescent health : official publication of the Society for Adolescent Medicine. 2007:41(1):99-101.

43. Shi Z, Lien N, Kumar BN, Holmboe-Ottesen G. Physical activity and associated socio-demographic factors among school adolescents in Jiangsu Province, China. Prev Med. 2006;43(3):218-21.

44. Tudor-Locke C, Ainsworth BE, Adair LS, Du S, Popkin BM. Physical activity and inactivity in Chinese school-aged youth: the China health and nutrition survey. International journal of obesity and related metabolic disorders : journal of the International Association for the Study of Obesity. 2003;27(9):1093-9.

45. Hertel T, Zhai F. Impacts of the Doha development agenda on China: the role of labor markets and complementary education reforms. In: Bank TW (ed.). Policy Research Working Paper. The World Bank; 2006;187(22):7727-37.

46. Hertel TW, Winters LA. Poverty impacts of a WTO agreement: synthesis and overview. In: Bank W (ed.), Washington, DC, 2005. [WWW document]. URL https://openknowledge.worldbank.org. (Accessed 11 Jan 2010).

47. National Bureau of Statistics of the Pepole's Republic of China, http://www. stats.gov.cn/. Accessed 12 Feb 2016.

48. Du SF, Wang HJ, Zhang B, Zhai FY, Popkin BM. China in the period of transition from scarcity and extensive undernutrition to emerging nutrition-related noncommunicable diseases, 1949-1992. Obes Rev. 2014;15(Suppl 1):8-15.

49. Zhai FY, Du SF, Wang ZH, Zhang JG, Du WW, Popkin BM. Dynamics of the Chinese diet and the role of urbanicity, 1991-2011. Obes Rev. 2014:15(Suppl 1):16-26.

50. Wang X, Liu QM, Ren YJ, Lv J, Li LM. Family influences on physical activity and sedentary behaviours in Chinese junior high school students: a crosssectional study. BMC Public Health. 2015;15:287.

51. Su C, Zhang B, Wang YF, Jia XF, Xue H, Wang HJ. Epidemics of overweight and obesity among growing childhood in China between 1997 and 2009: impact of family income, dietary intake, and physical activity Dynamics. Chin Med J. 2015;128(14):1879-86.

52. Morrissey $\mathrm{J}$, Janz KF, Letuchy EM, Francis SL, Levy SM. The effect of family and friend support on physical activity through adolescence: a longitudinal study. The international journal of behavioral nutrition and physical activity. 2015;12:103

53. Feng W, Gu B, Cai Y. The end of China's one-child policy. Stud Fam Plan. 2016;47(1):83-6.

54. Edwards MJ, Jago R, Sebire SJ, Kesten JM, Pool L, Thompson JL. The influence of friends and siblings on the physical activity and screen viewing behaviours of children aged 5-6 years: a qualitative analysis of parent interviews. BMJ Open. 2015:5(5):e006593.

55. Zang JJ, Ng SW. Age, period and cohort effects on adult physical activity levels from 1991 to 2011 in China. Int J Behav Nutr Phys Act. 2016:13:40.

56. Bleeker M, Beyler N, James-Burdumy S, Fortson J. The impact of playworks on boys' and girls' physical activity during recess. The Journal of school health. 2015:85(3):171-8.

57. Adams SA, Matthews CE, Ebbeling CB, et al. The effect of social desirability and social approval on self-reports of physical activity. Am J Epidemiol. 2005;161(4):389-98.

58. Sallis JF, Saelens BE. Assessment of physical activity by self-report: status, limitations, and future directions. Res Q Exerc Sport. 2000;71(Suppl 2):1-14.

59. Zhang J, Chaaban J. The economic cost of physical inactivity in China. Prev Med. 2013:56(1):75-8. 